\title{
Infezioni da H.pylori. Diagnosi: ill ruolo del gastroenterologo
}

\author{
Claudia Guatti -Zuliani \\ Servizio di Endoscopia Digestiva - Arcispedale Santa Maria Nuova - Reggio Emilia
}

\section{SUMMARY}

Diagnosis of $H$. pylori infection: the role of gastroenterologist

Helicobacter pylori (H. pylori) infection can be diagnosed by invasive techniques requiring endoscopy and biopsy (histological examination, rapid urease test, culture) and by non invasive techniques (serology, urea breath test, detection of $\mathrm{H}$. pylori antigen stool specimen). At present, no single test can be absolutely relied upon to detect colonization by $\mathrm{H}$. pylori and a combination of two tests is recommended if feasible. Nevertheless, in routine dayto-day clinical practice $H$. pylori diagnosis is often by a single test and consequently the choice of the more suitable test is even more important. Choosing among them is not easy, and several issues need to be considered, such as the clinical situation, (i.e. present symptoms and past medical history, age of patients, if it is first diagnosis or follow-up after treatment..), sensitivity and specificity of the test, the cost-effectiveness of the testing strategy, the availability of the test, the local expertise .

Since the "Ideal test" is not relied, the gastroenterologist or clinician as well, has the important role of deciding which test to employ. A good knowledge of advantages and vantages of each test is so necessary to make the best choose as possible.

\section{RELAZIONE}

Qual'è da ritenersi attualmente il ruolo del gastroenterologo, o del clinico più in generale, nell'ambito della diagnostica dell'infezione da Helicobacter pylori?

Da quando nel 1983 è avvenuto l'isolamento colturale dell'allora noto come Campylobacter pyloridis da parte di Marshall e Warren (1), sono state sviluppate successivamente molte tecniche diagnostiche per individuare la presenza dell'Helicobacter pylori. Ai giorni nostri abbiamo a disposizione un'ampia scelta di esami, di tipo sia invasivo sia non invasivo, da poter applicare nella pratica clinica quotidiana, ma nessuna delle tipologie testé citate é singolarmente in grado di stabile con assoluta certezza la presenza dell'H.pylori, tanto che lo stesso Gruppo di studio Europeo sull'H. pylori (EHPSG) riunitosi a Maastricht nel 2000 ha raccomandato, se possibile, l'esecuzione combinata di due test diagnostici (2).

Questa raccomandazione, resta però spesso inapplicata ed inapplicabile in quanto, nella pratica clinica, ci si trova nella necessità di dover ricorrere ad un unico test diagnostico.

Questa necessità implica quindi una scelta la quale risulta essere decisamente un punto critico. Sono molti gli elementi da prendere in considerazione i quali guidano il clinico nella sua scelta: in primis la situazione clinica del paziente a partire dall'età, dai sintomi e dalla sua storia clinica in generale; quindi, va fatto un attento distinguo se siamo di fronte ad una diagnosi primaria o ad un controllo post terapia eradicante e valutata l'utilità di informazioni aggiuntive che ci possono venire date dal test (es. informazioni sullo stato della mucosa gastrica, antibiogramma..).

Non va neanche trascurata l'esperienza del Centro, la disponibilità del test ed la rapidità con cui vogliamo avere i risultati, così come serve una valutazione economica in termini di cost-effectiveness. Altri aspetti da considerare sono poi la probabilità pre-test di infezione e le caratteristiche dei singoli test in termini di sensibilità e specificità.

Ciascun test diagnostico a nostra disposizione presenta i suoi vantaggi ed i suoi svantaggi ed è proprio dal bilanciamento degli uni e degli altri che deriva la sua applicazione clinica e tutta una serie di implicazioni cliniche.

Nella trattazione che segue ci si propone pertanto non solo di parlare in generale degli esami diagnostici di maggior impiego clinico, ma anche di presentare i rispettivi vantaggi e svantaggi commentandone alcune applicazioni/implicazioni cliniche conseguenti.

Il primo gruppo comprende i test invasivi, cioè quelli che necessitano del prelievo bioptico e quindi dell'esame endoscopico: istologia, test rapido all'ureasi, coltura; il secondo gruppo invece, è rappresentato dagli esami non invasivi: sierologia, breath test, ricerca antigene fecale.

\section{Istologia}

L'esecuzione dell'esame istologico, prevede una prima fase caratterizzata dall'indagine endoscopica con prelievo bioptico ad opera del gastroenterologo endoscopista, a cui segue la fase di allestimento del preparato istologico, la sua colorazione e la lettura del vetrino da parte dell'anatomo patologo.

\section{Vantaggi}

- l'esame istologico, non si limita a ricercare 
1'H.pylori, ma analizza anche la mucosa gastrica dandoci quindi informazioni sul danno infiammatorio od eventualmente degenerativo che si accompagna;

- i vetrini una volta preparati vengono conservati e possono essere disponibili per analisi retrospettive.

\section{Svantaggi}

- è un esame invasivo;

- è necessario un campionamento bioptico corretto, sia per sede sia per quantità di materiale prelevato, onde ottenere informazioni adeguate ed affidabili;

- è necessaria una colorazione adeguata nell'allestimento del vetrino per non incorrere in errate interpretazioni;

- è opportuno che l'anatomo patologo incaricato della lettura dei preparati istologici abbia una certa esperienza in materia;

- complessivamente è un esame costoso;

- non ci permette di ottenere informazioni in tempi rapidi.

I problemi, tanto quello del campionamento bioptico quanto quello della colorazione del preparato istologico, sono legati alla densità non uniforme con cui l'H. pylori colonizza lo stomaco.

Sebbene alcuni autori ritengano che, qualora l'istologia venisse adoperata unicamente per ricercare l'infezione, una singola biopsia dall'angulus gastrico sarebbe di per sé sufficiente ad assicurare una buona sensibilità diagnostica (3), attualmente, specie se si intende classificare la gastrite secondo il Sydney System $(4,5)$, è consigliabile l'esecuzione di due prelievi bioptici all'antro e due al corpo-fondo per ottenere una sensibilità diagnostica dell'esame istologico del 90-95\%.

Questo tipo di campionatura é particolarmente raccomandato in presenza di atrofia e metaplasia intestinale a livello dell'antro gastrico, in caso di recente terapia con farmaci antisecretivi e nel post terapia eradicante. Tali condizioni infatti possono portare ad una riduzione della densità batterica ed ad una modificazione dei siti di colonizzazione, con il corpo ed il fondo gastrico più infetti rispetto all'antro $(6,7)$.

L'H. pylori può essere già rivelato in sezioni colorate convenzionalmente con Ematossilina Eosina, ma tale metodica non è sempre agevole e comunque richiede osservazioni ad elevato ingrandimento ed anatomo patologi particolarmente esperti; inoltre, le difficoltà di identificazione aumentano se la densità del batterio non è elevata.

Altre colorazioni hanno mostrato una maggiore sensibilità come la Warthin Starry, che amplifica le dimensioni del batterio determinando una deposizione di granuli d'argento sulla superficie del germe, ma è una metodica gravata da difficoltà tecniche, costi elevati, possibilità di artefatti e lunghi tempi d'esecuzione; o come la Giemsa modificata, che per le sue caratteristiche di precisione, semplicità di esecuzione e basso costo, é preferita ad altre specie in caso di ridotta densità del germe.

Dallo studio di Laine L. et al. (8), in cui sono state confrontate le colorazioni Ematossilina Eosina, Genta e Giemsa con l'esame colturale, la colorazione di Giemsa ha riportato valori di sensibilità attorno al $90 \%$.

Accanto alle colorazioni esistono poi delle metodiche morfologiche più sofisticate, quali la microscopia elettronica e a scansione, la immunoistochimica con anticorpi monoclonali e la ibridizzazione di DNA in vitro, in grado di accrescere la specificità dell'esame ma che, per gli alti costi e il ricorso ad apparecchiature sofisticate, sono utilizzate solo a scopi di ricerca in pochi Centri diagnostici. Nella pratica corrente, la tipica morfologia del batterio e la sua distribuzione, sono elementi già sufficienti per una sua corretta identificazione.

\section{Test rapido all'ureasi}

Questo test sfrutta la proprietà dell'H.pylori di produrre l'enzima ureasi con cui scinde l'urea.

Un frammento bioptico della mucosa gastrica viene messo a contatto con degli elementi contenenti urea, i quali possono essere costituiti da un brodo oppure da un gel di agar o da un supporto di carta; in presenza del germe e quindi dell'ureasi, l'urea contenuta in tali supporti viene idrolizzata.

L'idrolisi dell'urea comporta la produzione di ioni ammonio e bicarbonato i quali innalzano il $\mathrm{pH}$ della soluzione, rivelato dal cambiamento di colore di un indicatore quale il rossofenolo. Pertanto, il viraggio dal colore giallo ( $\mathrm{pH}$ 6.8) al colore rossofenolo $(\mathrm{pH} 8.4)$, indica la positività del test ovvero la presenza dell' $H$. pylori nel frammento bioptico in esame.

Il risultato del test si può osservare già dopo 20 minuti, addirittura spesso il viraggio della colorazione è pressoché immediato, considerando però che in alcuni casi, i tempi di positivizzazione possono essere molto più lenti, da 60 minuti fino addirittura a 24 ore.

\section{Vantaggi}

- permette una diagnosi rapida;

- di per sé il test all'ureasi è un esame poco costoso e di facile esecuzione, se non si considera la necessità di dover eseguire sul paziente un esame endoscopico.

\section{Svantaggi}

- è un test comunque invasivo; 
- sebbene si esegua un prelievo bioptico, con il test all'ureasi non si ottengono informazioni sullo stato della mucosa gastrica;

- il test risente della bassa densità batterica;

- il sangue interferisce con l'affidabilità del test;

- terapie con farmaci antisecretivi interferiscono con l'affidabilità del test.

Il problema della bassa densità batterica non sembra inficiare l'efficacia diagnostica del test rapido all'ureasi nel paziente mai trattato, ove viene riportata una sensibilità tra 1 ' $80-95 \%$ ed una specificità del 95-100\% (9), ma diventa invece problema rilevante in caso di controllo post trattamento eradicante: qui si assiste ad una marcata riduzione della sensibilità con cadute addirittura dal $90 \%$ al $60 \%$ (10).

E' stato calcolato che per avere un test positivo sono necessari almeno 104 microrganismi, densità che può non essere raggiunta in alcuni pazienti (11).

Anche utilizzando un numero maggiore di materiale bioptico si è visto che non aumenta la sensibilità del test ma solo la velocità di reazione dello stesso (12). Alcune differenze di velocità di reazione sono state anche evidenziate tra test che usano supporti diversi: il Pyloritek ${ }^{\circledR}$, che utilizza supporto di carta, letto ad 1 ora ha riportato sensibilità e specificità comparabili a quelle del CLOtest $^{\circledR}$, il quale utilizza gel di agar come substrato, letto a 24 ore (13).

Dalla clinica sappiamo come la scoperta dell' $H$. pylori abbia rivoluzionato la gestione della malattia peptica: dimostrare la presenza del germe e la successiva terapia, ha contribuito alla netta riduzione dei casi di ulcere gastriche e duodenali comprese quelle sanguinanti. Pertanto, quando si fa diagnosi endoscopica di ulcera, la possibilità di testare nel più breve tempo possibile l'eventuale presenza dell'H.pylori, per poter impostare subito la terapia, farebbe del test rapido all'ureasi il test più indicato ed auspicabile.

Problemi però sono segnalati in letteratura nel caso di ulcere attivamente sanguinanti: la sensibilità del test all'ureasi passa infatti da un 90-95\% ad un $43-73 \%$ in corso di emorragia digestiva (14-16). Inizialmente, si era ipotizzata una possibile interferenza tra il sangue che contamina il campione bioptico ed il substrato utilizzato, spiegazione successivamente smentita (17).

Di recente, è stata invece dimostrata la capacità di alcuni fattori del plasma umano di uccidere l' $H$. pylori in vitro, capacità che potrebbe verificarsi anche in vivo (18).

Test all'ureasi, falsamente negativi, si possono ottenere anche in pazienti con acloridria, o in coloro che fanno uso di farmaci antisecretivi come gli inibitori della pompa protonica o gli
anti-H2 antagonisti. Infatti, con l'incremento del $\mathrm{pH}$ del lume gastrico, si raggiungono valori di $\mathrm{pH}$ estremamente elevati vicino al germe in maniera tale che l'H. pylori viene distrutto dall'azione della sua stessa ureasi $(12,19)$.

\section{Coltura}

La crescita di colonie di batteri ricurvi e gramnegativi che producono ureasi, ossidasi e catalasi è la dimostrazione assoluta dell'infezione da H.pylori. Per tale motivo, l'esame colturale dovrebbe essere il test di riferimento nella diagnosi di infezione.

Essendo tuttavia metodica lunga e laboriosa, che richiede personale dedicato ed accorgimenti speciali di laboratorio, l'esame colturale diventa, di fatto, poco accurato e pertanto improponibile quale "gold standard" (20).

Proprio in quanto metodica laboriosa, è importante per un clinico conoscerne i vantaggi e svantaggi per poter correttamente indicarne il suo utilizzo.

\section{Vantaggi}

- il test offre la possibilità di eseguire un antibiogramma, ossia testare la sensibilità del germe ai vari antibiotici;

- il test offre la possibilità di eseguire una tipizzazione dei ceppi batterici.

\section{Svantaggi}

- il test risente della qualità e quantità del campionamento bioptico;

- il test risente delle modalità di trasporto, conservazione ed allestimento del materiale;

- una recente terapia antibiotica o con antisecretivi o bismuto, può interferire con i risultati del test;

- i risultati del test sono legati all'esperienza dell'operatore che lo esegue;

- sono necessari tempi lunghi prima di avere i risultati dell'esame;

- il test ha un costo elevato.

Per definizione, la specificità dell'esame colturale è assoluta dal momento che l'isolamento del batterio in vitro consente la sua precisa identificazione, mentre la sua sensibilità dipende essenzialmente dal numero, dalla sede di prelievo, dal trasporto e dalla processazione in laboratorio dei campioni bioptici e varia dal 75 al 94\%.

Per la fattibilità del test un solo prelievo bioptico dall'antro dovrebbe, teoricamente, essere sufficiente, dal momento che anche pochi batteri sono in grado di moltiplicarsi rapidamente sui terreni di coltura (21); un secondo prelievo bioptico dal corpo gastrico è comunque raccomandato nei casi in cui si vuole controllare l'eradicazione o se si sospetta una metaplasia intestinale dell'antro oppure se il paziente ha assunto di recente farmaci in grado di ridurre la carica batterica, quali inibitori di pompa protonica, antibiotici o bismuto. 
Sicuramente il motivo principale per cui si ricorre alla coltura, è la possibilità di poter effettuare l'antibiogramma: questa informazione aggiuntiva risulta utile di fronte al fallimento di più terapie eradicanti o se si opera in aree ad elevata antibiotico resistenza.

\section{Sierologia}

L'organismo umano entrando in contatto con l' $H$. pylori genera una risposta anticorpale che è sia di tipo locale (cioè di mucosa) mediata da IgA, sia sistemica (cioè nel sangue periferico) caratterizzata da un precoce e rapido picco di IgM e successivamente da una risposta IgG sostenuta, quest'ultima utilizzata dai test sierologici sia nell'adulto che nel bambino.

Partendo dal concetto che, una volta acquisita, l'infezione da $H$. pylori cronicizza e solo raramente si eradica in modo spontaneo, ne consegue che la presenza di anticorpi anti-H. pylori è sempre indicativa di infezione attiva in individui che non sono mai stati trattati in precedenza.

Questa risposta anticorpale costante, può essere quindi utilizzata a scopo diagnostico con la ricerca nei liquidi biologici delle immunoglobuline specifiche come indice di infezione.

Le metodiche più standardizzate e note si riferiscono a valutazioni sul siero, ma non dobbiamo dimenticare che anticorpi anti $H$. pylori si possono rinvenire, e sono stati anche proposti a scopo diagnostico, nella saliva, nei secreti gastrici e nelle urine.

I test sierologici vengono schematicamente distinti in test di "laboratorio", quando eseguiti con strumentazioni ad hoc presso laboratori attrezzati, e test "ambulatoriali", quando sono effettuati "di fronte" al paziente (i cosidetti "near patient test" o "office" test) fornendogli un risultato immediato. Accanto a questi vi sono poi i test sierologici che ricercano specificatamente anticorpi anti-Cag A ed anti-VacA, cioè contro le proteine prodotte dai ceppi più virulenti di H.pylori. L'associazione fra anticorpi anti- CagA e patologia gastroduodenale severa (malattia ulcerosa e cancro gastrico) aveva fatto ipotizzare l'uso di questi test sierologici quali markers di patogenicità (22); tuttavia non essendo stata questa associazione definitivamente confermata, l'impiego di questi test nella pratica clinica è ancora oggetto di discussione.

Per determinare il titolo anticorpale sierico sono state suggerite ed impiegate diverse metodiche: test di fissazione del complemento, emoagglutinazione, agglutinazione batterica, immunofluorescenza, ELISA (enzyme linked immunoabsorbent assay, cioè metodica immunoenzimatica), Western blotting, immunocromatografia.

Attualmente la metodica ELISA è la più usata nei vari laboratori, ed in commercio esistono numersi kit ELISA la cui principale differenza è rappresentata dall'antigene: alcuni sono semipurificati, altri sono una miscela di antigeni purificati provenienti da uno o più ceppi.

\section{Vantaggi}

- è un test non invasivo;

- è un test "globale", cioè capace di valutare la presenza dell'infezione indipendentemente dalla sua topografia di colonizzazione gastrica; pertanto, a differenza dei test invasivi basati sulla biopsia endoscopica, la sierologia non è soggetta ad errori di campionamento;

- non risente di eventuali interferenze con farmaci antisecretivi e antibiotici;

- permette di ottenere la risposta in tempi brevi;

- è esame poco costoso e di facile esecuzione.

\section{Svantaggi}

- manca una standardizzazione delle preparazioni antigeniche, vi sono infatti numerosi kit sierologici in commercio;

- questo è conseguenza della variabilità di risposta anticorpale tra gli individui, nessun antigene dell'H.pylori viene infatti riconosciuto da tutti i sieri;

- dai due aspetti precedenti consegue che è difficile il confronto tra risultati emessi da laboratori diversi;

- vi può essere un numero elevato di risultati indeterminati, alcuni kit arrivano addirittura ad un $12 \%$ di risultati indeterminati;

- non è possibile controllare in tempi brevi l'efficacia della terapia eradicante.

Numerosi sono gli studi effettuati per la valutazione dell'accuratezza diagnostica dei kit diagnostici (23).

In linea di massima la performance dei test "di laboratorio" è generalmente superiore a quella dei test "ambulatoriali", ma risulta comunque bassa: una meta-analisi di 21 studi con kit ELISA "di laboratorio" riporta una sensibilità dell' $85 \%$ ed una specificità del 79\% (24), così come dall'analisi di 588 sieri mediante 16 diversi kit ELISA viene riportata un'accuratezza complessiva del 78\% (range: $68-83 \%$ ) (25).

Occorre anche considerare che i valori di sensibilità e specificità sono estremamente variabili anche per lo stesso kit da un Centro all'altro. Questo può dipendere da una differente prevalenza di infezione nelle popolazioni esaminate, dall'età dei soggetti, dalla concomitante somministrazione di farmaci (es. FANS), dalla coesistenza di altre malattie, ma anche dal ceppo o dai ceppi di H.pylori prevalenti nella popolazione in esame. Gli studi di immunoblotting hanno infatti dimostrato, che esistono delle differenze sia qualitative 
che quantitative del pattern di risposta anticorpale in soggetti appartenenti a diverse aree geografiche e ciò sottolinea l'importanza sia del ceppo di H.pylori sia dei metodi di preparazione dell'antigene utilizzati nei kit sierologici.

In caso di successso della terapia eradicante, il titolo anticorpale diminuisce e può arrivare a negativizzarsi. Il tempo di sieroconversione è tuttavia variabile e, da una media di latenza di 6 mesi, in alcuni casi si può arrivare anche a 2-3 anni (26). Complessivamente la sierologia è test a bassa accuratezza che trova attualmente principale applicazione nel campo epidemiologico; nella clinica può essere talora impiegato nella diagnosi primaria, per esempio in caso di pazienti in terapia antisecretiva nei quali, come vedremo in seguito, gli altri test non invasivi sono poco affidabili, ma sicuramente non è test da impiegarsi nel post terapia.

\section{Urea Breath test (UBT)}

Questo test si basa sull'idrolisi, indotta dall'attività ureasica dell'H. pylori, di urea isotopicamente marcata con $\mathrm{C}^{14}$ (radioattivo) o $\mathrm{C}^{13}$ (stabile non radioattivo) e sulla conseguente formazione di ammonio ed ${ }^{13 / 14} \mathrm{CO}_{2}$ (27). Dopo esser stata assorbita attraverso le pareti gastriche, la $\mathrm{CO}_{2}$ marcata passa nel pool dei bicarbonati plasmatici e viene quindi espirata. La presenza nel respiro di ${ }^{13 / 14} \mathrm{CO}_{2}$ dopo somministrazione orale di urea marcata, equivale pertanto a dimostrare la presenza di ureasi nella cavità gastrica e quindi la presenza di H.pylori. Se invece l'H.pylori è assente, l'urea marcata non viene idrolizzata (le cellule dell'organismo umano non possiedono attività ureasica) ed è eliminata con le feci.

Una volta che l'aria espirata è stata raccolta, all'interno di provette o sacchetti appositi, questa verrà analizzata.

Le tecniche di rilevazione della $\mathrm{CO}^{2}$ marcata sono diverse a seconda dell'isotopo adoperato. Mentre la ${ }^{14} \mathrm{CO}_{2}$ viene misurata con un beta counter o scintillografo, strumento generalmente presente in numerose istituzioni mediche, la ${ }^{13} \mathrm{CO}_{2}$ viene misurata con uno spettrometro di massa atomica, strumento sofisticato disponibile in pochi Centri. La differenza tra ${ }^{14} \mathrm{C}$-UBT e ${ }^{13} \mathrm{C}$-UBT non consiste unicamente nelle tecniche di misurazione, ma anche nel fatto che se il primo espone ad una dose di radioattività, il secondo è totalmente non radioattivo e pertanto è stato preferito nell'applicazione clinica. Nella trattazione si farà riferimento al ${ }^{13} \mathrm{C}$-UBT anche se molte delle considerazioni che seguiranno sono applicabili anche al ${ }^{14} \mathrm{C}$-UBT.

Per una corretta applicazione dell'urea breath test va rispettata una precisa metodologia frutto di importanti lavori di validazione.

I protocolli presentati da questi lavori differiscono sostanzialmente nei seguenti aspetti: dose di urea da somministrare, tipo di pasto standard usato per rallentare lo svuotamento gastrico, tempi di raccolta dell'espirato e valori di cut-off. Nella tabella 1 sono schematizzate le caratteristiche dei tre protocolli più diffusi per l'adulto:

1' "europeo" (28), 1' "americano" (29) ed il "tedesco" (30); e del protocollo per l'età pediatrica (31).

\section{Vantaggi}

- è un test non invasivo;

- è un test "globale" cioè è capace di valutare la presenza dell'infezione;

- indipendentemente dalla sua topografia di colonizzazione gastrica, pertanto a differenza dei i test invasivi basati sulla biopsia endoscopica, l'UBT non è soggetto ad errori di campionamento;

- è un test riproducibile anche in Centri diversi;

- è di facile esecuzione;

- può essere utilizzato nei bambini;

- permette di verificare i risultati di una terapia eradicante dopo breve tempo;

è un test con alta sensibilità e specificità sia prima sia dopo una terapia eradicante

\section{Svantaggi}

- la mancanza di ampia disponibilità della strumentazione: lo spettrofotometro di massa è infatti apparecchio costoso presente solo in alcuni Centri;

- come conseguenza di quanto detto sopra, l'urea breath test è esame che presenta ancora un costo significativo;

- vi può essere interferenza con farmaci antisecretivi ed antibiotici e pertanto, prima di eseguire l'UBT, viene consigliato un periodo di cosiddetto "wash out" (astenersi dall'assumere antibiotici nel mese precedente ed antisecretivi nei 15 giorni precedenti l'esame);

- vi può essere interferenza con farmaci che accelerano lo svuotamento gastrico, pertanto,

Tabella I Protocolli di validazione del ${ }^{13} \mathrm{C}$-urea breath test

\begin{tabular}{|llllll|}
\hline Protocollo & Autore Anno & Dose ${ }^{13}$ C-urea & Pasto standard & Tempo raccolta & Cut-off \\
\hline Europeo & Logan R 199I & $100 \mathrm{mg}$ & $100 \mathrm{ml} \mathrm{lipidi}$ & $30 \mathrm{~min}$ & $5 \%$ \%० \\
\hline Americano & Klein PD 1996 & $125 \mathrm{mg}$ & $237 \mathrm{ml} \mathrm{lipidi}$ & $30 \mathrm{~min}$ & $2.4 \% \circ$ \\
\hline Tedesco & Labenz J 1996 & $75 \mathrm{mg}$ & $100 / 200 \mathrm{ml} \mathrm{ac.} \mathrm{citrico}$ & $30 \mathrm{~min}$ & $4 \% \circ$ \\
\hline Età pediatrica & Bazzoli F 2000 & $50 \mathrm{mg}$ & Pasto lipidico & $30 \mathrm{~min}$ & $3.5 \% \circ$ \\
\hline
\end{tabular}


si consiglia di sospendere i farmaci procinetici prima dell'esame;

- è stata segnalata anche una scarsa affidabilità del test in certe condizioni patologiche quali l'uremia ed in caso di situazioni anatomiche modificate come dopo interventi di chirurgia a livello gastrico.

L'accuratezza del urea breath test, sia nella diagnosi primaria sia dopo terapia eradicante, è oramai indiscussa; dai lavori in letteratura emergono ottime percentuali di sensibilità e specificità.

Dalla media dei risultati dei lavori riassunti nelle tabelle 2 e 3 , i valori di sensibilità e specificità dell'urea breath test sono rispettivamente del $97.6 \%$ e $97 \%$ pre trattamento e del $95 \%$ e $97 \%$ dopo terapia eradicante (32-34). Anche per quanto riguarda l'età pediatrica con il protocollo messo appunto da Bazzoli $\mathrm{F}$ et al. (31) i valori di sensibilità e specificità nella diagnosi primaria si assestano attorno al $97 \%$.

Tabella 2 Valori di sensibilità e specificità dell'urea breath test nella diagnosi primaria

\begin{tabular}{lccc}
\hline Autore-Anno & Pazienti & Sensibilità (\%) & Specificità(\%) \\
\hline Klein I996 & 463 & 95 & 88 \\
\hline Thijs 1996 & 105 & 100 & 100 \\
\hline Dominguez 1997 & 80 & 100 & - \\
\hline Epple 1997 & 126 & 96 & 100 \\
\hline Moayyedi 1997 & 222 & 98 & 96 \\
\hline Alì I998 & 119 & 100 & 96 \\
\hline Leodolter I998 & 40 & 100 & 100 \\
\hline Leodolter 1999 & 553 & 93 & 98 \\
\hline Vaira I999 & 501 & 95 & 98 \\
\hline van der Hulst 1999 & 544 & 95 & 94 \\
\hline Gisbert 2000 & 53 & 100 & 100 \\
\hline Savarino 2000 & 354 & 99 & 97 \\
\hline & 3160 & 97,6 & 97 \\
\hline
\end{tabular}

Tabella 3 Valori di sensibilità e specificità dell'urea breath test dopo terapia eradicante

\begin{tabular}{lccc}
\hline Autore-Anno & Pazienti & Sensibilità (\%) & Specificità(\%) \\
\hline Klein 1996 & 173 & 95 & 95 \\
\hline Epple 1997 & 126 & 96 & 100 \\
\hline Alì 1998 & 63 & 98 & 92 \\
\hline Vaira 1999 & 107 & 90 & 99 \\
\hline Vaira 2000 & 235 & 91 & 99 \\
\hline Savarino 2000 & |31 & 100 & 97 \\
\hline & $\mathbf{8 3 5}$ & $\mathbf{9 5}$ & $\mathbf{9 7}$ \\
\hline
\end{tabular}

L'urea breath test è vantaggioso come test di verifica dopo terapia eradicante, non solo per la sua accuratezza diagnostica, ma anche per la rapidità con cui tale accuratezza viene raggiunta: sono infatti sufficienti solo 4 settimane dal termine della terapia affinché il test si negativizzi in caso di successo terapeutico, e questo si verifica alla stessa maniera in tutti i soggetti, a differenza dell'estrema variabilità vista per la sierologia. Tale confronto viene schematizzato nel grafico 1.

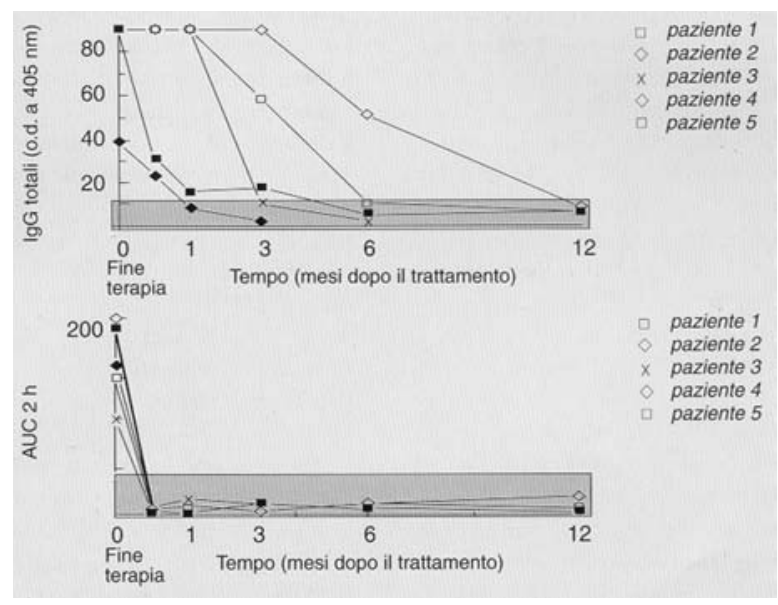

Grafico I Andamento della risposta della sierologia (in alto) e dell'urea breath test (in basso) in caso di successo della terapia eradicante

Va segnalato che uno dei maggiori svantaggi dell'urea breath test, legato alla scarsa diffusione dello spettrofotometro di massa, oggi può essere agevolmente aggirato se si instaura una rete che prevede l'invio di tutti i campioni di aria raccolti in provette o nei sacchetti appositi dai Centri più piccoli verso i Centri di riferimento che provvederanno alla lettura dei campioni e a fornire i risultati.

\section{Ricerca dell'antigene fecale (HpSA)}

L'identificazione diretta degli antigeni del batterio nelle feci rappresenta la più recente metodica non invasiva a disposizione del clinico. Il test si basa sul metodo EIA (immuno enzimatico) che consiste nel porre il campione fecale contenente l'eventuale antigene in pozzetti ove si trovano adsorbiti degli anticorpi policlonali specifici (35). In presenza dell'antigene si sviluppa una reazione di colore di tipo qualitativo il cui valore viene confermato da uno spettrofotometro.

\section{Vantaggi}

- è un test "globale" cioè è capace di valutare la presenza dell'infezione indipendentemente dalla sua topografia di colonizzazione gastrica, pertanto, a differenza dei i test invasivi basati sulla biopsia endoscopica, la ricerca dell'antigene fecale non è soggetta ad errori di campionamento;

- è di facile esecuzione;

- ha un basso costo;

- è utilizzabile nei bambini anche molto piccoli;

- permette di verificare i risultati di una terapia eradicante dopo breve tempo;

- è un test ad alta sensibilità e specificità.

\section{Svantaggi}

- esattamente come per l'urea breath test, anche 
in questo caso vi può essere interferenza con farmaci antisecretivi ed antibiotici e pertanto viene consigliato un periodo di cosiddetto di "wash out" (astensione dall'assunzione di antibiotici nel mese precedente ed antisecretivi nei 15 giorni precedenti l'esame);

- viene segnalata la possibile interazione con altri antigeni batterici.

Gli studi sull'accuratezza del test fecale riportano percentuali di sensibilità e specificità comparabili con quelle dell'urea breath test per quanto riguarda la prima diagnosi di infezione, attorno ad un $92-93 \%$, mentre nel post terapia eradicante esiste una certa variabilità di risultati da parte dei vari autori (32-34, 36-40).

Tali differenze potrebbero in parte dipendere dal fatto che il test usato come "gold standard" con il quale confrontare l'HpSA non è stato lo stesso in tutti gli studi. Nelle tabelle 4 e 5 sono schematizzati i risultati di revisioni sistematiche su lavori che hanno studiato l'accuratezza del test raggruppati per periodi di pubblicazione.

Tabella 4 Sensibilità e specificità della ricerca dell'antigene fecale $(H p S A)$ nella diagnosi primaria: revisioni sistematiche Revisioni sistematiche Pazienti Sensibilità (\%) Specificità(\%)

\begin{tabular}{lccc}
\hline |999-2000 & 2924 & $\mathbf{9 3 . 1}$ & $\mathbf{9 2 . 8}$ \\
\hline 1999-giugno 200| & $34 \mid 9$ & $\mathbf{9 3 . 2}$ & $\mathbf{9 3 . 2}$ \\
\hline Gisbert 200| (25 studi) (37) & 4769 & 92 & 92
\end{tabular}

Tabella 5 Sensibilità e specificità della ricerca dell'antigene fecale $(H p S A)$ dopo terapia eradicante: revisioni sistematiche e singoli lavori

\begin{tabular}{|c|c|c|c|}
\hline $\begin{array}{l}\text { Revisioni } \\
\text { sistematiche/studi }\end{array}$ & Paz. & Sensibilità (\%) & Specificità (\%) \\
\hline |999-giugno 200| & 332 & 92.1 & 87.6 \\
\hline \multicolumn{4}{|c|}{ ( 2 test come gold standard) } \\
\hline |999-giugno 200| & 790 & 88.8 & 87.3 \\
\hline \multicolumn{4}{|c|}{ (UBT come gold standard) } \\
\hline $\begin{array}{l}\text { Gisbert 200I } \\
\text { (25 studi) (37) }\end{array}$ & 2078 & 88 & 92 \\
\hline Manes 200I (38) & 106 & 87.5 & 95.7 \\
\hline Leodolter 200I (39) & 113 & 76.2 & 93.3 \\
\hline Vaira $2002(40)$ & 84 & 94 & 97 \\
\hline
\end{tabular}

In un recente lavoro italiano (41), su 100 pazienti dispeptici $H$. pylori positivi è stata confrontata la ricerca dell'antigene fecale con l'urea breath test nel post trattamento, utilizzando come "gold standard" l'esame istologico su biopsie eseguite in corso di gastroscopia dopo 4 settimane dal termine della terapia. La tabella 6 sintetizza i risultati dello studio: l'urea breath test si è dimostrato più accurato della ricerca dell' antigene fecale nel post terapia.
Tabella 6 Confronto tra ${ }^{13} \mathrm{C}-\mathrm{UBT}$ ed HpSA nel post trattamento (pazienti $n^{\circ}: 100$ )

\begin{tabular}{lcc}
\hline & I3C-UBT & HpSA \\
\hline SENSIBILITA' (\%) & 91 & 87 \\
\hline SPECIFICITA' (\%) & 98 & 88 \\
\hline VALORE PREDITTIVO POSITIVO (\%) & 95 & 69 \\
\hline VALORE PREDITTIVO NEGATIVO (\%) & 97 & 96 \\
\hline ACCURATEZZA COMPLESSIVA & 97 & 88 \\
\hline
\end{tabular}

Anche il Gruppo di Studio Europeo sull'H. pylori (EHPSG) si è espresso riguardo $i$ test da utilizzare nella diagnostica non invasiva dell'infezione da H. pylori. In caso di diagnosi primaria, si raccomanda l'utilizzo allo stesso modo o dell'urea breath test o della ricerca dell'antigene fecale. Quando invece vanno controllati i risultati della terapia eradicante si consiglia l'urea breath test, a meno che non si debba ricorrere ad un test invasivo per una precisa indicazione clinica all'esame endoscopico.

Infine, l'EHPSG sottolinea che la ricerca dell'antigene fecale è al momento ancora da ritenersi un'alternativa, qualora l'urea breath test non fosse disponibile (2).

Da questa carrellata sui test diagnostici per l'infezione da H.pylori emerge, come al momento non abbiamo a disposizione quello che potremmo definire il "Test diagnostico ideale", cioè quel test che raggruppa assieme tutta una serie di caratteristiche positive quali:

- elevata accuratezza in ogni contesto clinico;

- riproducibilità;

- non invasività;

- facilità di esecuzione;

- basso costo;

- rapidità nel fornire i risultati;

- disponibilità presso ogni Istituzione Sanitaria. Purtroppo, ognuno dei test attualmente applicabili nella pratica clinica, soddisfa solo alcuni degli aspetti sopraelencati mentre è più carente in altri; questo lascia al gastroenterologo, o più in generale al clinico, l'importante compito di scelta del test più appropriato al contesto clinico $o$ in grado di soddisfare alcune specifiche richieste.

Ovviamente tutto questo è possibile solo conoscendo vantaggi e svantaggi, o se vogliamo dire "pregi e difetti" di tutti gli esami diagnostici a disposizione.

\section{BIBLIOGRAFIA}

1. Marshall BJ, Warren JR. Unidentified curved bacilli in the stomach of patients with gastritis and peptic ulceration. Lancet 1984; 1: 1311-5.

2. Malfertheiner P and the European Helicobacter Pylori Study Group (EHPSG) Current concepts in the management of Helicobacter pylori infection- The Maastricht 2-2000 Consensus Report. Aliment Pharmacol Ther 2002; 16: 167-80.

3. Genta RM, Graham DY. Comparison of biopsy sites 
for the histopathological diagnosis of Helicobacter pylori infection: a topographic study of $\mathrm{H}$. pylori density and distribution. Gastrointest Endosc 1994; 40: 342-5.

4. Price AB. The Sydney System: histological division. J Gastroenterol Hepatol 1991; 6: 209-22.

5. Dixon MF, Path FRC, Genta R, Yardley JH, Correa P et al. Classification and grading of gastritis. The Updated Sydney System. Am J of Surg Pathol, 1996; 20: 1161-81.

6. Stolte M, Bethke B. Elimination of Helicobacter pylori under treatment with omeprazole. Z Gastroenterol 1990 ; 28: 271-4.

7. Satoh K, Rimura K et al. Biopsy sites suitable for the diagnosis of Helicobacter pylori infection and the assessment of the extent of atrophic gastritis. Am J Gastroenterol 1998; 93: 569-73.

8. Laine L, Lewin DN, Naritoku W, Cohen H. Prospective comparison of H\&E, Giemsa, and Genta stains for the diagnosis of Helicobacter pylori. Gastrointest Endosc 1997; 45: 463-7.

9. Midolo P, Marshall BJ Accurate diagnosis of Helicobacter pylori: urease tests. Gastroenterol of North Am 2000; 29: 871-8.

10. Nishikawa K, Sugiyama T, Kato M et al. A prospective evaluation of new rapid urease tests before and after eradication treatment of Helicobacter pylori in comparison with histology culture and C-urea breath test. Gastrointest Endosc 2000; 51: 164-8.

11. Megraud F. Advantages and disavantages of current diagnostic tests for the detection of Helicobacter pylori. Scand J Gastroenterol 1996; 21 (suppl 215): 57-62.

12. Laine L, Chun D, Stein C et al. The influence of size or number of biopsies on rapid urease test results: a prospective evaluation. Gastrointest Endosc 1996; 43: 49-53.

13. Puetz T, Vakil N, Dunn B et al. The Pyloritek test and CLOtest: accuracy and incremental cost analysis. Am J Gastroenterol 1997; 92: 254-7.

14. Archimandritis A, Tzivras M, Sougioultzis S et al. Rapid urease test is less sensitive than Hystology in diagnosing Helicobacter pylori infection in patients with non-variceal upper gastrointestinal bleeding $\mathrm{J}$ Gastroenterol Hepatol 2000; 15: 369-73.

15. Lee JM, Breslin NP, Fallon C, O’Morain CA. Rapid urease tests lack sensitivity in Helicobacter pylori diagnosis when peptic ulcer disease presents with bleeding. Am J Gastroenterol 2000; 95: 1166-70.

16. Tu TC, Lee CL, Wu CH et al. Comparison of invasive and noninvasive tests for detecting Helicobacter pylori infection in bleeding peptic ulcers. Gastrointest Endosc 1999; 49: 302-6.

17. Laine L, Sidhom O, Emani S, Estrada R, Cohen H. Effect of blood on rapid urease testing of gastric mucosal biopsy specimens. Gastrointest Endosc 1998; 47: $141-43$.

18 Houghton J, Ramamoorthy R, Panda H, Dhirmalani $\mathrm{R}$, Kim KH. Human plasma is directly bacteriocidal against Helicobacter pylori in vitro, potentially explaining the decreased detection of Helicobacter pylori during acute upper GI bleeding. Gastrointest Endosc 2002; 55: 11-6.

19 Lerang F, Moum B, Mowinckel P et al. Accuracy of seven different tests for the diagnosis of Helicobacter pylori infection and the impact of $\mathrm{H}_{2}$ receptor antagonists on test results. Scand J Gastroenterol 1998; 33: 364-9.

20. Goodwin CS, Mendall MM, Northfield TC. Helicobacter pylori infection. Lancet 1997; 349: 265-9.

21. Megraud F. Diagnosis of Helicobacter pylori infec- tion. Scand J Gastroenterol 1996; 31: 44-6.

22 Parsonnet J, Friedman GD, Orantreigh $\mathrm{N}$ et al. Risk of gastric cancer in people with Cag A positive or Cag A negative Helicobacter pylori infection. Gut 1997; 40: 297-301.

23. Vaira D, Holton J, Menegatti M et al. Blood tests in the management of Helicobacter pylori infection. Gut 1998; 43 (suppl 1): S39-S46.

24. Loy CT, Irwig LM, Katelaris PH, Talley NJ. Do commercial serological kits for Helicobacter pylori infection differ in accuracy? A meta-analysis. Am J Gastroenterol 1996; 91:1138-44

25. Stevens M, Livsey S, Swann R, Rathborne B. Evaluation of sixteen EIAs for the detection of antibodies to Helicobacter pylori. London Department of Health 1997; 1-46.

26. Cutler AF, Prasad VM. Long term follow up of Helicobacter serology after successful eradication of Helicobacter pylori. Am J Gastroenterol 1996; 91: 85-8.

27. Perri F, Goos Y, Hiele M et al. The urea breath test: a non-invasive clinical tool for detecting Helicobacter pylori infection. It J Gastroenterol 1995; 27: 5-63.

28. Logan RPH, Dill S, Bauer FE et al. The European ${ }^{13} \mathrm{C}$ urea breath test for detection of Helicobacter pylori. Eur J Gastroenterol Hepatol 1991; 3: 915-21.

29. Klein PD, Malaty HM, Martin RF et al. Non invasive detection of Helicobacter pylori infection in clinical practice: the ${ }^{13} \mathrm{C}$-urea breath test. Am J Gastroenterol 1996; 91: 690-4.

30. Labenz J, Borsch G, Peitz U et al. Validity of a novdel biopsy urease test (HUT) and a simplified ${ }^{13} \mathrm{C}$-urea breath test for diagnosis of $\mathrm{H}$. pylori infection and estimation of the severity of gastritis Digestion 1996; 57: 391-7.

31. Bazzoli F, Cecchini L, Corvaglia L et al. Validation of the ${ }^{13} \mathrm{C}$-urea breath test for the diagnosis of Helicobacter pylori infection in children: a multicenter study. Am J Gastroenterol. 2000 Mar;95(3): 646-50.

32. Vaira D, Holton J, Menegatti M, et al. Review article: invasive and non-invasive tests for Helicobacter pylori infection. Aliment Pharmacol Ther 2000; 14 (suppl 3): 13-22.

33. Vaira D \& Vakil N. Blood, urine, stool, breath, money, and Helicobacter pylori. Gut 2001; 48: 287-89.

34. Vaira D, Gatta L, Ricci C \& Miglioli M. Review article: diagnosis of Helicobacter pylori infection. Aliment Pharmacol Ther 2002; 16 (suppl 1): 16-23.

35. Vaira D, Malfertheiner P, Megraud F et al. Diagnosis of Helicobacter pylori infection with a new non -invasive antigen-based assay. Lancet 1999; 354: 30-3.

36. Mitchell $\mathrm{H}$ and Megraud F. Epidemiology and diagnosis of Helicobacter pylori infection. Helicobacter 2002; 7 (suppl 1): 8-16.

37. Gisbert JP and Pajares JM. Diagnosis of Helicobacter pylori infection by stool antigen determination: a systematic review. Am J Gastroenterol 2001; 96: 2829-38.

38. Manes G, Balzano A, Iaquinto G et al. Accuracy of stool antigen test in posteradication assessment of Helicobacter pylori infection. Dig Dis Sci 2001; 46: 2440-44.

39. Leodolter A, Agha-Amiri K, Peitz U et al. Validity of a Helicobacter pylori stool antigen assay for the assessment of $\mathrm{H}$. pylori status following eradication therapy. Eur J Gastroenterol Hepatol 2001; 13: 673-6.

40. Vaira D, Vakil N, Menegatti M et al. The stool antigen test for detection of Helicobacter pylori after eradication therapy. Ann Intern Med 2002; 136: 280-7.

41. Biliardi C, Biagini R, Dulbecco P et al. Stool antigen assay $(\mathrm{HpSA})$ is less reliable than urea breath test for post-treatment diagnosis of Helicobacter pylori infection. Aliment Pharmacol Ther 2002; 16: 1733-38. 